\title{
Knowledge and practices regarding doffing of Personal Protective Equipment among frontline health care workers during COVID-19
}

\author{
Vidanapathirana T \\ Accident \& Emergency Department, District General Hospital, Matara
}

\begin{abstract}
Background :Personal Protective Equipment (PPE) has become a widely talked topic due to the recent Corona virus pandemic which was declared a Public Health Emergency of International Concern in March, 2020. The objective of this study was to assess the knowledge and practices regarding doffing of Personal Protective Equipment among front line health care workers in District General Hospital Matara, Sri Lanka.

Method: A total of 279 front line health care workers attached to COVID-19 units in District General Hospital Matara were included in the study consisting of all categories of health care workers.

Results: Majority were nurses (55.2\%; $n=154)$ while 33.7\% ( $n=33.7)$ were medical officers. Only 68.1\% ( $n=190)$ have received training on how to wear PPE before appointing to work in COVID-19 units. Overall knowledge on types of PPE anddoffing practices was $>80 \%$ and $>60 \%$ respectively. There was a significant positive association between participating in a training session on PPE and the knowledge on correct doffing practices $\left(X^{2}=7.081\right.$; $p=0.012)$. A significant number of medical officers did not wear impermeable gowns $(p=0.001)$, gloves $(p=0.048)$, face shields $(p=0.008)$, goggles $(p<0.001)$ and boots $(p<0.001)$ compared to the other categories taken together. Majority of the participants (46.2\%) stated 'shortage of PPE' as the reason for inadequate use of PPE.

Conclusion: Quality and timely training on PPE and continuous availability of PPE are important in reducing transmission of infectious diseases.
\end{abstract}

Key words: COVID-19,Personal Protective Equipment (PPE), health care workers,knowledge and practices Introduction

\begin{abstract}
$A^{s}$ s described by The Occupational Safety and Health Administration(OSHA), personal protective equipment(PPE)are specially designed clothing or equipment used to prevent or minimize contact with hazardous substances in a work place ${ }^{1}$. In health care settings, PPE is an essential component to control of infection and safety of workers. PPE acts as a physical barrier and protects the health care worker ( $\mathrm{HCW}$ ) from getting exposed to hazardous materials such as blood, body fluids etc. It prevents transmission of communicable diseases from one person to another. Some of the PPE used in health care settings are face masks, gloves, gowns and goggles. According to OSHA, it is the responsibility of the employer to make appropriate PPE available for employees, ensure that employees are trained on proper handling of PPE and make sure that the employees are properly using the PPE2.
\end{abstract}

\section{Corresponding Author:}

Thushara Vidanapathirana

Email:tvidanapathirana@yahoo.com

(iD) https://orcid.org/0000-0002-1107-801X
PPE has become an important and widely discussed topic due to the recent coronavirus outbreak (COVID-19) which was declared aPublic Health Emergency of International Concern (PHEIC) by the World Health Organization (WHO) on 11th March, 2020. Availability and proper usage of PPE became a major concern around the world. WHO and the Center for Disease Control (CDC) released guidelines on rational use of PPE and how to handle shortage of PPE3,4.

In order to get the optimal effectiveness of using PPE, donning (putting on) and doffing (taking off) of PPE should be error-free. Doffing process is relatively more important in preventing transmission to infections than the donning process 5 .

Studies conducted in many parts of the world to assess the knowledge and practices regarding PPE among the health care personnel have found striking results.

A study conducted in a hospital in Chicago to assess the doffing practices of $107 \mathrm{HCWs}$, using an observation check list, found that $21 \%$ removed the gloves incorrectly and $65 \%$ removed the gown incorrectly while $26 \%$ removed the mask incorrectly. Overall incorrect 
doffing practices was $90 \%$. But the study did not reveal any significant difference in doffing practices among different categories of HCWs $(p=0.79) 6$.

Another study conducted in University of Pittsburgh, video recorded the donning and doffing of PPE among $65 \mathrm{HCWs}$, after obtaining their consent. Even knowing they are being videotaped, the percentage of incorrect doffing practices was as high as $79.2 \%{ }^{7}$.

There were published data of few studies conducted in South East Asian region but, as far as author knows, there has not been any published work on this topic conducted in Sri Lanka.

A Korean study conducted in a tertiary care hospital recruiting 29 participants; physicians and nurses, revealed that frequent incorrect techniques were observed during removing respirators (79.2\%), removing shoe covers $(65.5 \%)$ and removing the hood $(41.3 \%)^{8}$.

As PPE is one of the important strategies in prevention of transmission of infectious diseases in health care settings, the assessment of proper usage of PPE among HCWs is an important need. In a situation where the whole world is overwhelmed with the COVID-19 pandemic, assessment of adherence to standard PPE handling protocols is a timely need.

This study was conducted to explore the level of knowledge, practices and training exposure related to proper usage of PPE among HCWs and to determine the associations of knowledge and practices regarding doffing of Personal Protective Equipment with job category and training exposure of the HCWs in a local setting. Though both donning and doffing of PPE are equally important in blocking transmission of infections, this study only looked in to doffing practices. The findings of this study are expected to be used in identifying the gaps in infection control activities. Moreover, the results will aid in planning the training activities for different categories of HCW. The ultimate intention of this study is to reduce the infection rate and thereby to reduce the mortality rate and stabilize the health care system of the country via rational usage of PPE.

\section{Methodology}

The study was conducted in District General Hospital Matara from September to October 2020. All categories of front line HCWs in District General Hospital Matara were included in the study. Those who were refrained from working in the front line due to a medical reason were excluded from the study. All participants were fluent in Sinhala language. A total of 281 HCWs were randomly recruited from each category proportionate to the size of each category. A pre tested self-administered questionnaire developed by the investigator consisting of socio-demographic data, data on training related to PPE and knowledge on doffing of PPE (as per Institutional guidelines used in District General Hospital Matara, WHO guidelines and CDC guidelines)was used for data collection. Pilot testing was done inTeaching Hospital Karapitiya. Questionnaire was adjustedaccording to the results of the pilot test. Data collection was done by the investigator.Data analysis was done using SPSS version 23 software. Permission to conduct the study was obtained from the Director of District General Hospital Matara. Informed written consent was obtained from the participants after explaining about the study.Questionnaires, information sheets and consent forms were in both Sinhala and English languages and the procedurewas explained in a convenient language for the participants. Ethical clearance was obtained from Ethics Review Committee, Faculty of Medicine, University of Peradeniya.

Results

\section{Socio-demographic details}

A total of 281 health care workers participated in the study. There were $2(0.71 \%)$ incompletely filled questionnaires; the response rate was $99.3 \%$. All participants were Sinhalese, Buddhists while majority (75.6\%; $n=211$ )were females. Age of the participants ranged between 29 to 58 years while the mean age was 40.32 years. Majority of the participants were nurses $(55.2 \%, n=154)$ followed by medical officers $(33.7 \%, \mathrm{n}=94)$, minor staff $(10 \%, \mathrm{n}=28)$ and matrons/ sisters $(1.1 \%, n=3)$.

\section{Details related to work as a front line HCW and the training obtained on PPE}

It was noted that $39.1 \%(n=109)$ have worked for 6 months or more in COVID-19 unitswhile the rest $(n=170,60.9 \%)$ have worked for less than 6 months. Only $68.1 \%$ ( $n=190$ ) have received training on how to wear PPE before appointing as a front line health care worker in COVID-19 wards. The number of training sessions the participantsparticipated varied from 1 to 10. Out of these 190 participants, majority (36.8\%, $\mathrm{n}=70$ ) had participated only 1 training session. Sixty four (33.7\%) and $33(17.4 \%)$ had received 2 and 3 training sessions respectively while the rest of the participants $(n=23,12.1 \%)$ were exposed to more than 3 training sessions. The training sessions comprised of demonstrations of handling PPE $(52.7 \%$, $n=147)$, lectures $(45.5 \%, n=127)$, videos $(41.2 \%$, $\mathrm{n}=115)$, hands on skill training $(28.3 \%, \mathrm{n}=79)$, workshops $(19 \%, n=53)$ and distribution of leaflets $(4.3 \%$, $\mathrm{n}=12$ ) where some training sessions included more than one methods mentioned above.

\section{Knowledge regarding PPE}

Knowledge on PPE used in health care settings for COVID-19 was as shown in table 1. 
Table 1: Knowledge regarding PPE

\begin{tabular}{|c|c|c|c|}
\hline \multirow{4}{*}{$\begin{array}{l}\text { Majority } \quad(n=193,69 \%) \\
\text { knew the correct order of } \\
\text { doffing of PPE. Out of that, }\end{array}$} & \multirow[t]{2}{*}{ PPE used in a health care setting for COVID-19 } & \multicolumn{2}{|l|}{ Yes } \\
\hline & & $\mathrm{n}$ & $\%$ \\
\hline & Impermeable gown & 234 & 83.9 \\
\hline & Gloves & 270 & 96.8 \\
\hline b3 & Face masks & 270 & 96.8 \\
\hline rs. In & Face shields & 255 & 91.4 \\
\hline nificant positive associa- & Goggles & 252 & 90.3 \\
\hline tion between participat- & Surgical hoods or caps & 258 & 92.5 \\
\hline ing in a training session on & Covered shoes and fluid resistant shoe covers or boots & 237 & 84.9 \\
\hline
\end{tabular}

When asked whether they think they are adequately equipped with PPE, 145 (52\%) said that they think they are wearing PPE adequately 'most of the times' while 115 (41.2\%) said they think they are properly equipped with correct doffing practices $\left(X^{2}=7.081 ; p=0.012\right)$. There was no significant association between job category andsatisfactory knowledge on order of doffing of PPE ( $p=0.586)$.

Knowledge on doffing practices was as shown in table 2. Majority gave the correct answer for all the practices assessed.

In the assessment

Table 2: Knowledge on doffing practices

to describe the source of information the participants used to obtain knowledge regarding proper usage of PPE,majority $\quad(n=262$, 93.9\%) stated they

\begin{tabular}{|l|l|l|l|}
\hline Doffing practices & $\begin{array}{l}\text { Correct } \\
\text { answer }\end{array}$ & $\mathbf{n}$ & $\%$ \\
\hline Hand rub should be used in between each step of doffing PPE & True & 237 & 84.9 \\
\hline Removed boots should be washed with soap and water & False & 207 & 74.2 \\
\hline Removed face shield/ goggles should be washed with 1\% TCL & True & 175 & 62.7 \\
\hline Should have a bath before going home & True & 275 & 98.6 \\
\hline PPE should be discarded in to a closed bin with a yellow colour bag & True & 263 & 94.3 \\
\hline PPE should be immediately removed after the procedure & True & 258 & 92.5 \\
\hline If you are leaving the clinical area, you should not remove the PPE & False & 265 & 95 \\
\hline
\end{tabular}

PPE 'all the time'. Only 5 (1.8\%) said that they 'never' think they are adequately equipped with PPE.

The reasons given by the participants for not wearing the PPE adequately were as shown in the table 5. One hundred and twenty nine (46.2\%) stated that they had a shortage of PPE supply at some point in time (Table 5). The participants were asked whether there was any shortage of PPE and the type of PPE which was out of stock (Figure 1 and Table 6).

rely on hospital

protocols. A reasonable number referred to WHO web site $(82.8 \%, n=231)$ and Ministry of Health web site $(69.5 \%, n=194)$.

Practices regarding Personal Protective Equipment The participants were asked about their practices regarding wearing PPE while working as a front line health care worker in COVID-19 units. More than 85\% were wearing all required PPE when in contact with the COVID-19 patients (Table 3).

\begin{tabular}{|c|c|c|c|}
\hline \multirow{9}{*}{$\begin{array}{l}\text { Association be- } \\
\text { tween job catego- } \\
\text { ryand wearing PPE } \\
\text { when in contact } \\
\text { with COVID-19 } \\
\text { patients is shown } \\
\text { in table } 4 \text {. Nurses, } \\
\text { sisters, matrons } \\
\text { and minor staff } \\
\text { were grouped to- } \\
\text { gether as 'other }\end{array}$} & \multicolumn{3}{|l|}{ Table 3: Practice of wearing PPE } \\
\hline & $\begin{array}{l}\text { PPE the participants used to wear always } \\
\text { when working in COVID-19 units }\end{array}$ & $\mathbf{n}$ & $\%$ \\
\hline & Impermeable gown & 239 & 85.7 \\
\hline & Gloves & 263 & 94.3 \\
\hline & Face masks & 274 & 98.2 \\
\hline & Face shield & 268 & 96.1 \\
\hline & Goggles & 249 & 89.2 \\
\hline & Surgical hood or cap & 253 & 90.7 \\
\hline & Covered shoes and fluid resistant shoe covers or boots & 238 & 85.3 \\
\hline
\end{tabular}
categories'. Medical officers

were wearing impermeable gowns $(p=0.001)$, gloves $(p=0.048)$, face shields $(0.008)$, goggles $(p=<0.001)$ and boots $(<0.001)$ significantly less than the health care workers in other categories (table 4). case of COVID-19 in Sri Lanka was reported on 27th January 2020; a 44 year old Chinese woman admitted to the National Institute of Infection Diseases. Since then the health sector of Sri Lanka took necessary measures to control the cases within the country by establishing COVID-19 units in certain hospitals. Therefore majority of the participants (36.2\%) had an experience of 6 months working in COVID-19 wards. According to this study, only $68.1 \%(n=190)$ had received a training on proper use of PPE before appointing to COVID-19 wards. This is not a satisfactory figure as it is evidenced that proper training on handling PPE reduces the risk of transmission of COVID-19 to HCW9, ${ }^{10}$. The study showed a significant positive association between participating in a training session on PPE and the knowledge on correct doffing practices $\left(X^{2}=7.081 ; p=0.012\right)$. A similar finding was obtained in an intervention study conducted in a tertiary care hospital in Beijing, China where there 
Table 4: Association between job category and wearing PPE

\begin{tabular}{|c|c|c|c|c|}
\hline Variable & Yes & No & Chi square valve df & $P$ value \\
\hline \multicolumn{5}{|c|}{ Wearing impermeable gown } \\
\hline Medical officers & $71(75.5 \%)$ & $23(24.5 \%)$ & 11.84 & 0.001 \\
\hline Other categories & $168(90.8 \%)$ & $17(9.2 \%)$ & 1 & \\
\hline \multicolumn{5}{|l|}{ Wearing gloves } \\
\hline Medical officers & $85(90.4 \%)$ & $9(9.6 \%)$ & 3.8 & 0.048 \\
\hline Other categories & $178(96.2 \%)$ & $7(3.8 \%)$ & 1 & \\
\hline \multicolumn{5}{|l|}{ Wearing masks } \\
\hline Medical officers & $94(100 \%)$ & $0(0 \%)$ & Fisher's exact test & 0.126 \\
\hline Other categories & $180(97.3 \%)$ & $5(2.7 \%)$ & & \\
\hline \multicolumn{5}{|l|}{ Wearing face shields } \\
\hline Medical officers & $86(91.5 \%)$ & $8(8.5 \%)$ & Fisher's exact test & 0.008 \\
\hline Other categories & $182(98.4 \%)$ & $3(1.6 \%)$ & & \\
\hline \multicolumn{5}{|l|}{ Wearing goggles } \\
\hline Medical officers & $72(76.6 \%)$ & $22(23.4 \%)$ & 23.64 & $<0.001$ \\
\hline Other categories & $177(95.7 \%)$ & $8(4.3 \%)$ & 1 & \\
\hline \multicolumn{5}{|c|}{ Wearing surgical cap or hood } \\
\hline Medical officers & $81(86.2 \%)$ & $13(13.8 \%)$ & 3.41 & 0.054 \\
\hline Other categories & $172(93 \%)$ & $13(7 \%)$ & 1 & \\
\hline \multicolumn{5}{|l|}{ Wearing boots } \\
\hline Medical officers & $66(70.2 \%)$ & $28(29.8 \%)$ & 25.75 & $<0.001$ \\
\hline Other categories & $172(93 \%)$ & $13(7 \%)$ & 1 & \\
\hline
\end{tabular}

was a very significant improvement in the post-test compared to the pre-test after an training programme on PPE $(p<0.001){ }^{11}$.

The current study revealed that more than $80 \%$ health care workers knew the types of PPE used in COVID-19 units while more than $70 \%$ had adequate knowledge on doffing practices. In contrast to this finding the studies conducted outside Sri Lanka revealed poor overall knowledge on doffing of PPE among health care workers $6,7,12$. This difference could have been due to the observation component included in most of these studies which revealed more reliable information. A study conducted in West Indies, Jamaica revealed that $90 \%$ nurses, $88 \%$ doctors and $70 \%$ medical technicians were very knowledgeable of universal precautions ${ }^{4}$. The present study revealed that a significant number of medical officers did not wear impermeable gowns $(p=0.001)$, gloves $(p=0.048)$, face shields $(0.008)$,
Table 5: Reasons given by the participants for not wearing PPE adequately

\begin{tabular}{|c|c|c|c|}
\hline Reason & \multicolumn{2}{|l|}{$\mathbf{n}$} & $\%$ \\
\hline \multirow[t]{2}{*}{ I was not knowledgeable enough } & Medical officers & 5 & \multirow[t]{2}{*}{5.4} \\
\hline & Other categories & 10 & \\
\hline \multirow[t]{2}{*}{ I was not trained properly } & Medical officers & 19 & \multirow[t]{2}{*}{24} \\
\hline & Other categories & 48 & \\
\hline \multirow[t]{2}{*}{ Due to lack of time } & Medical officers & 2 & \multirow[t]{2}{*}{2.9} \\
\hline & Other categories & 6 & \\
\hline \multirow[t]{2}{*}{ My negligence } & Medical officers & 15 & \multirow[t]{2}{*}{11.8} \\
\hline & Other categories & 18 & \\
\hline \multirow[t]{2}{*}{ I do not consider it as a risk } & Medical officers & 6 & \multirow[t]{2}{*}{3.2} \\
\hline & Other categories & 3 & \\
\hline \multirow[t]{2}{*}{ Due to shortage of PPE/ other materials } & Medical officers & 39 & \multirow[t]{2}{*}{46.2} \\
\hline & Other categories & 90 & \\
\hline \multirow[t]{2}{*}{ Due to controversial health messages } & Medical officers & 14 & \multirow[t]{2}{*}{10.8} \\
\hline & Other categories & 16 & \\
\hline
\end{tabular}

goggles $(p<0.001)$ and boots $(p<0.001)$ compared to nurses and minor staff taken together. Contrary to the findings of this study, a study conducted in Tamil $\mathrm{Nadu}^{13}$ revealed that doctors had significantly good compliance towards using appropriate PPE compared to nurses and technicians ( $p=0.0001)$.

As highlighted by this study, the shortage of PPE supply was a huge problem in many parts of the world 
Table 6

\begin{tabular}{|l|l|l|}
\hline Type of PPE on shortage & $\mathbf{n}$ & $\mathbf{\%}$ \\
\hline Impermeable gown & 174 & 62.4 \\
\hline Gloves & 7 & 2.5 \\
\hline Face masks & 170 & 60.9 \\
\hline Face shields & 115 & 41.2 \\
\hline Goggles & 102 & 36.6 \\
\hline Surgical hoods or caps & 77 & 27.6 \\
\hline $\begin{array}{l}\text { Covered shoes and fluid } \\
\text { resistant shoe covers or boots }\end{array}$ & 101 & 36.2 \\
\hline
\end{tabular}

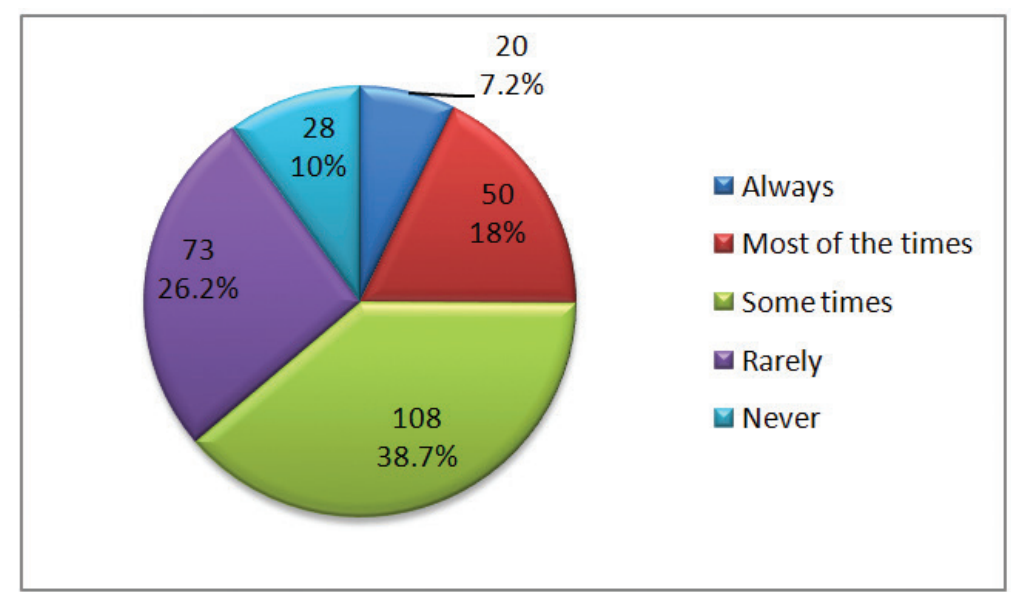

Figure 1: Presence of a shortage of PPE at any point in time

15,16 and high demand for face masks made the face masks the most scarce PPE 16 as revealed by this study.

However, one limitation of this study was that the practices of doffing of PPE were assessed by asking them about their practices. But these practices would have been better assessed if the study included an observation component.

In conclusion it is very important that the relevant authorities take steps to give quality training on PPE to all categories of HCW including doctors, before appointing them to high risk units. Furthermore the hospital authorities should have plans to make PPE available without a shortage for high risk units throughout the pandemic period.
United States Department of Labour. https://www.osha.gov/aboutosha

2. OSHA Field Safety and Health Manual.; 2011.

3. World Health Organization. Rational Use of Personal Protective Equipment for Coronavirus Disease (COVID-19): Interim Guidance.; 2020.

4. Using Personal Protective Equipment (PPE). Center for Disease Control. Published 2020. https://www.cdc. gov/coronavirus/2019-ncov/hcp/using-ppe.html

5. COVID-19 Infection Prevention and Control - Application of PPE during COVID-19 Version 2.; 2020.

6. Phan LT, Maita D, Mortiz DC, et al. Personal protective equipment doffing practices of healthcare workers. J Occup Environ Hyg. 2019;16(8):575-581. doi:10.1080/15459624.2019.1628350

7. Kang JH, O'Donnell JM, Colaianne B, Bircher N, Ren D, Smith KJ. Use of personal protective equipment among health care personnel: Results of clinical observations and simulations. Am J Infect Control. 2017;45(1):17-23. doi:10.1016/j.ajic.2016.08.011

8. Lim SM, Cha WC, Chae MK, Jo IJ. Contamination during doffing of personal protective equipment by healthcare providers. Clin Exp Emerg Med. 2015;2(3):162-167. doi:10.15441/ ceem.15.019

9. Gagneux-Brunon A, Pelissier C, Gagnaire J, et al. SARS-CoV-2 infection: advocacy for training and social distancing in healthcare settings. J Hosp Infect. Published online 2020. doi:10.1016/j.jhin.2020.08.001

\section{References}

1. Occupational Safety and Health Administration.
10. Houghton C, Meskell P, Delaney $\mathrm{H}$, et al. Barriers and facilitators to healthcare workers' adherence with infection prevention and control (IPC) guidelines for respiratory infectious diseases: A 
rapid qualitative evidence synthesis. Cochrane Database Syst Rev. 2020;4(4):1-55. doi:10.1002/14651858.CD013582

11. Tan, Wenhui; Ye, Yuying; Yang, Yijing; Chen, Zhu; Yang, Xinglong; Zhu, Caizhong; Chen, Dong; Tan, Junyuan; Zhen C. Whole-Process Emergency Training of Personal Protective Equipment Helps Healthcare Workers Against COVID-19. J Occup Environ Med. 2020;62(6):420-423.

12. Kwon JH, Burnham CAD, Reske KA, et al. Assessment of Healthcare Worker Protocol Deviations and Self-Contamination during Personal Protective Equipment Donning and Doffing. Infect Control Hosp Epidemiol. 2017;38(9):1077-1083. doi:10.1017/ ice.2017.121

13. P. A. AL, Jennifer H. G, Stanly A. M, Paul CM. A study on personal protective equipment use among health care providers, Tamil Nadu. Int J Community Med Public Heal. 2018;5(5):1771-1774. doi:10.18203/23946040.ijcmph20181380

14. Vaz K, Mcgrowder D, Alexander-Lindo R, Gordon L, Brown P, Irving R. Knowledge, Awareness and Compliance with Universal Precautions among Health Care Workers at the University Hospital of the West Indies, Jamaica. Vol 1.; 2010.

15. Mandrola J. COVID-19 and PPE: Some of us will die because of the shortage. Recenti Prog Med. 2020;111(4):183. doi:10.1701/3347.33175

16. Burki T. Global shortage of personal protective equipment. Lancet. 2020;20:785-786. doi:10.1016/S1473-3099(20)30501-6 